\title{
A Escolha dos Líderes e a Compreensão Emocional na Educação Pré-Escolar
}

\section{Choosing the leaders and Emotional Understanding in Preschool Education}

\author{
Glória Franco*, Sara Jardim** \\ * Universidade da Madeira, ** Secretaria Regional da Educação
}

\begin{abstract}
Resumen
Este estudo teve por objectivo perceber a importância que as variáveis pessoais, as relações interpessoais e a compreensão emocional poderão ter na escolha de um líder em crianças a frequentar o Ensino Pré-Escolar. Participaram quarenta e cinco crianças, $46,7 \%$ do sexo feminino e 53,3\% do sexo masculino, com idades entre os três e seis anos, da Região Autónoma da Madeira (RAM). Os resultados mostraram que o sexo da criança e ter irmãos, bem como, a preferência social, o impacto social, o estatuto social influenciam a escolha para líder. Da compreensão emocional só a regulação emocional mostrou estar associada a esta escolha.

Palavras chave: Liderança; Compreensão Emocioanl; Educação Pré-Escolar.
\end{abstract}

\begin{abstract}
The aim of this study is to understand the importance that personal variables, interpersonal relationships, and emotional understanding may have in choosing a leader in children attending Pre-School. Forty-five children, 46.7\% female and 53.3\% males, aged between three and six years, from the Autonomous Region of Madeira (RAM) participated. The results showed that the child's gender and having siblings, as well as, social preference, social impact, and social status influence the choice for leader. From emotional understanding only emotional regulation is associated with this choice.
\end{abstract}

Keywords:Leadership; Emotional understanding;

Preschool Education.

\section{Introdução}

A liderança está intimamente ligada ao sucesso nos vários domínios da nossa vida (Bento, 2008), pelo que se torna cada vez mais imperativo estimular esse tipo de comportamento em crianças pequenas, assim como dar-lhes ferramentas para que descubram os seus talentos sociais e emocionais. No entanto, a liderança em crianças pequenas é um tema pouco investigado (Lee, Recchia, \& Shin, 2005, Rechia, 2011, Morda \& Waniganayake, 2010).

Ao longo dos anos, diversos autores têm tentado definir a liderança na infância, assim como, os tipos de líderes existentes e de que forma uns se destacam dos outros. Este tem sido um trabalho algo infrutífero, pois parece não se encontrar um consenso sobre estes conceitos, aparecendo liderança associado a várias características dos sujeitos.
Apesar dos fatores situacionais e das atividades e composição do grupo afetarem o aparecimento de certos indivíduos como líderes, nas crianças parecem exibir certas características que contribuem para a possibilidade de emergirem como líderes num grupo (Scharf \& Mayseless, 2009).

Segundo alguns estudos (Popper \& Mayseless, 2003; Popper, Mayseless, \& Castelnovo, 2000, Popper, Amit, Gal, Mishkal-Sinai, \& Lisak, 2004), os indivíduos com mais segurança estão melhor preparados do que os outros para se tornarem líderes sociais por causa da internalização positiva e representações cuidadas do eu e dos outros significativos. Fukada, Fukada \& Hicks (1994) na sua investigação citam Segal et al. (1987), em que o estilo social é um atributo da liderança para eles. As aptidões interpessoais são, também, consideradas por alguns autores, características típicas dos líderes. Para Scharf \& Mayseless (2009) é esperado que os líderes tenham um modelo positivo de si e dos outros e isso inclui preocupar-se com os outros e ter boas aptidões interpessoais, o que está em linha com a noção de que os líderes são capazes e estão dispostos a contribuir para o grupo. O líder deve possuir estratégias pró-sociais de gestão de conflitos, tais como querer harmonizar em vez de se vingar. Com a prática destes objetivos/metas e estratégias nas relações diádicas com os amigos, as crianças tornam-se proficientes nas competências interpessoais prósociais que são úteis em grandes grupos. Vários investigadores (Farmer \& Rodkin, 1996; Hawley, 2002; Pakaslahti \& Keltikangas-Jarvinen, 2001; Rodkin, Farmer, Pearl, \& VanAcker, 2000, Scharf \& Mayseless, 2009) mostram nos seus estudos com crianças, que a maioria dos líderes é sociável e agradável.

$\mathrm{O}$ estatuto sociométrico tem-se também mostrado importante para a capacidade de liderança (Löfdahl, 2006; Perez et al., 1982; Fukada, Fukada \& Hicks, 1997; Fukada et al.,1994; Chen, Rubin, \& Sun, 1992; Dodge, 1983). Fukada et al. (1994, 1997) mostraram que as crianças em idade pré-escolar do grupo sociométrico mais elevado têm papéis de liderança com mais frequência do que os do grupo sociométrico mais baixo.

Outros atributos são também identificados como contribuindo para a escolha dos líderes, como sejam, a ordem de nascimento (Hardy et al., 1978, citados por Fukada et al., 1994), a autoestima (Nath \& Scriven, 1981, citados por Fukada et al., 1994), o estatuto no grupo de 
brincadeira (Fukada, 1991, 1992, citado por Fukada et al., 1994), a capacidade de decisão e camaradagem (Kitano, 1983).

Segundo Hensel (1991), um pré-requisito de liderança e competências pró-sociais é conseguir ver as siuações da perspetiva dos outros. No seu estudo as crianças eram encorajadas a ver as coisas de diferentes perspetivas, uma vez de assumir uma perspetiva visual de um indivíduo é mais facilmente compreendido e mais concreto do que assumir a perspetiva emocional individual de outra pessoa. Em segundo lugar, as crianças foram envolvidas nas suas próprias experiências e foi discutido como se sentiam nessas situações. A autora achou importante que as crianças entendessem os seus próprios sentimentos antes de tentarem entender os dos seus colegas de sala. Assim, estas discussões centraram-se na identificação do problema, descrição do que sentiam, rotulagem/ nomeação/ etiquetagem do sentimento e identificação de novas situações que poderiam evocar o mesmo sentimento. Em terceiro lugar, cada criança tinha de inferir os sentimentos dos outros. Depois das questões e sentimentos terem sido identificados, as crianças criaram possíveis soluções através de brainstorming. As questões levantadas pelo professor ajudaram a que as crianças considerassem mais do que uma perspetiva no desenvolvimento de soluções. Após as possíveis soluções terem sido listadas e representadas pelas crianças, foram discutidas várias soluções, vantagens e desvantagens e os sentimentos das crianças acerca de cada um deles. No final do estudo, as observações efetuadas, sugeriram que as crianças que estavam mais despertas para os sentimentos dos colegas, encontravam, com maior frequência, maneiras de ajudar os seus companheiros, assim como, conseguiam articular, com maior clareza, o seu entendimento pela perspetiva dos outros e eram mais escolhidas escolhidas para líderes

Assim a questão que direcionou este estudo foi a de compreender de que modo o conhecimento emocional é importante na escolha para líder.

\section{Método}

\section{Participantes}

A amostra é constituída por dois grupos de crianças do pré-escolar, com quarenta e cinco crianças, vinte e um indivíduos do sexo feminino $(46,7 \%)$ e vinte e quatro do sexo masculino $(53,3 \%)$, com idades compreendidas entre os três e os seis anos de idade, de duas escolas básicas do $1^{\circ}$ ciclo com pré-escolar da Região Autónoma da Madeira (RAM), uma em meio rural e a outra em meio urbano.

\section{Instrumentos}

Foram utilizados três instrumento: 1) um Questionário Sociodemográfico; 2) um teste sociométrico; 3) o Test of Emotion Comprehension (TEC) de Pons, Harris e Rosnay (2004), na sua versão portuguesa Teste de Compreensão das Emoções de Roazzi, Dias, Minervino, Roazzi e Pons (2008).

O questionário sociodemográfico serviu para recolher dados relativos: a) identificação do respondente; b) dados pessoais da criança; c) situação familiar e d) dados Sociais.

O teste sociométrico tinha três questões direcionadas para a escolha de pares e líderes. A saber: a) De todos os teus colegas, diz-me três que gostarias de levar para o passeio ao Parque de Santa Catarina (quem gostas mais)?; b) De todos os teus colegas, diz-me três que não gostarias de levar para o passeio ao Parque de Santa Catarina (quem gostas menos)?; c) Se tivesses de escolher, de todos os teus colegas, diz-me três que escolherias para ser o/a chefe da sala (quem achas que é o melhor chefe)?

Para se realizar este teste, todas as crianças participantes do estudo foram fotografadas individualmente e foram mostradas as fotografias para ver se cada criança sabia identificar os seus colegas. $\mathrm{Na}$ resposta a cada uma das perguntas, a criança indicava o nome de três colegas de sala, apontando para a respetiva fotografia.

Para obtermos o estatuto social, usámos o método utilizado por Peceguina, Santos e Daniel (2008), que por sua vez se basearam no trabalho de Coie et al.. Este método tem como alicerce as frequências absolutas de nomeações positivas e negativas, que cada criança recebeu. Após a obtenção destas frequências, estas são transformadas em resultados estandardizados (z scores) que representam as medidas LM (like most - gosta mais) e LL (like least - gosta menos). Com estes dois resultados estandardizados são calculados os resultados da preferência social $(\mathrm{P}=\mathrm{LM}-\mathrm{LL})$ e do impacto social (I = LM + LL). Segundo Coie e colaboradores (1982, citados por Alves, 2006), o impacto social ou "visibilidade", pode ser definido como a soma das escolhas/LM e das rejeições/LL recebidas pelo indivíduo. Já a preferência social ou "popularidade" corresponde à diferença entre o número de escolhas e o número de rejeições alcançadas pelo indivíduo. Para formarmos o sistema de classificação do estatuto social, utilizámos um sistema de classificação bidimensional dos estatutos, resultando em cinco grupos com base na distribuição normal: 1) crianças populares - $\mathrm{P}>1.0$, $\mathrm{LM}>0$ e $\mathrm{LL}<0$; 2) crianças rejeitadas $-\mathrm{P}<-1.0, \mathrm{LL}>0$ e $\mathrm{LM}<0$; 3) crianças negligenciadas $-\mathrm{I}<-1.0$ e frequência absoluta de nomeações positivas $=0 ; 4)$ crianças controversas - I> 1.0, LM e LL > 0; 5) crianças médias todas as crianças que não são identificadas nas restantes categorias. Assim, para que uma criança possa ser classificada dentro dum destes estatutos, as condições postas para cada estatuto terão de acontecer em paralelo, isto é, os valores de P, I, LM e LL deverão encontrar-se dentro dos limites apresentados.

O Test of Emotion Comprehension (TEC) tem como objetivo estudar o desenvolvimento da compreensão emocional em crianças, distinguindo nove componentes: 1) reconhecer as emoções, através da expressão facial (Reconhecimento emocional); 2) compreender as causas situacionais externas de emoções (Causas externas); 3) compreender a relação entre desejo e emoções (Desejos); 4) compreender a relação entre crenças e emoções (Crenças); 5) compreender o impacto das lembranças na emoção (Lembranças); 6) compreender os efeitos de distração (Regulação); 7) conhecer a diferença entre 
emoções verdadeiras e evidentes (Esconder emoções); 8) compreender a possibilidade de experimentar emoções mistas (Emoções misturadas); 9) compreender a relação de sensações de culpa e de errado (Expressões morais). Foi a partir das respostas de uma amostra de crianças inglesas, que as propriedades psicométricas do TEC foram obtidas, sendo prioridade a análise da estrutura fatorial e da consistência interna do instrumento (alfa de Cronbach =0,67). O TEC permite-nos medir a compreensão destes nove componentes em crianças com idades entre os três e os doze anos.

\section{Procedimentos}

Foram pedidas as devidas autorizações à Direção Regional da Administração Educativa; às Direções das Escolas e aos encarregados de educação. Foram garantidos os princípios éticos do anonimato, da confidencialidade e da livre participação das crianças.

O Questionário Sociodemográfico foi o primeiro a ser preenchido por todos os encarregados de educação.

As crianças começaram por realizar o Teste Sociométrico, em que eram mostradas as fotografias de todas as crianças da sala de atividade, exceto a da criança que estava a responder às questões por nós apresentadas e era pedido para escolher três colegas de sala de acordo com a questão colocada.

Seguiu-se o TEC, a cada criança foi explicado que deveria ouvir com atenção as instruções dadas pela voz do computador e que se não percebesse alguma coisa deveria dizer para que a instrução/pergunta fosse repetida. Também, foi dito que não deveria ter pressa em responder a cada pergunta e que a resposta deveria ser sempre pensada e escolhida por ela.

Os instrumentos foram aplicados às crianças numa só sessão, por um investigador experimentado, e numa sala com boas condições e sem distratores, tendo tido a duração média de 40m.

Para o tratamento de dados, utilizámos o SPSS (software aplicativo usado para análise estatística, acrónimo de Statistical Package for the Social Sciences), versão 23 , que nos ajudou a fazer a análise estatística dos dados recolhidos para este estudo.

\section{Resultados}

Após termos verificado que não existia normalidade e homogeneidade na distribuição da escolha do chefe para as variáveis Idade; Sexo; Escolas/Residência; Tem irmãos?; Número de irmãos mais velhos; Número de irmãs (feminino); Número de irmãos (masculino); Frequentou o infantário?, fizemos o teste não paramétrico Mann-Whitney (cf. tabela 1). Pode-se observar que destas só o ter irmãos tem influência, sendo que $\mathrm{U}=117,500, \mathrm{p}=, 023$. Já o facto de ter ou não irmãos mais velhos não é importante para se ser escolhido como líder, nem o ter irmãos de outro sexo. Também a variável Idade da criança, assim como o Sexo, a Escola/Residência ou mesmo a frequência ou não do infantário não se mostraram relevantes na escolha para líder.
Tabela 1

Testes Mann-Whitney para verificar a influência nas Notas $T$ chefe das variáveis sociodemográficas

\begin{tabular}{|c|c|c|c|c|}
\hline & $\mathrm{M}-\mathrm{W}$ & Signif. & Média & Moda \\
\hline Idade da Criança & 192,500 & ,887 & 1,7333 & \\
\hline Sexo da Criança & 196,500 & ,204 & & 1,00 \\
\hline Escolas/Residência & 245,000 & ,908 & & 2,00 \\
\hline Tem irmãos? & 117,500 &, 023 & & 1,00 \\
\hline $\begin{array}{l}\text { Número de irmãos } \\
\text { mais velhos }\end{array}$ & 179,000 & ,116 & ,5778 & \\
\hline $\begin{array}{l}\text { Número de irmãs } \\
\text { (feminino) }\end{array}$ & 159,500 & ,389 & ,2889 & \\
\hline $\begin{array}{l}\text { Número de irmãos } \\
\text { (masculino) }\end{array}$ & 179,000 & ,095 & ,4667 & \\
\hline $\begin{array}{l}\text { Frequentou o } \\
\text { infantário? }\end{array}$ & & ,421 & &, 00 \\
\hline
\end{tabular}

Ao observarmos a preferência social correlacionada com a escolha, a rejeição e o chefe (cf. Tabela 2), encontrámos três correlações significativas $(r=, 602 ; r=$ ,606; $\mathrm{r}=348$ ), as duas primeiras boas e a última moderada, isto é, à medida que a preferência social aumenta, aumentam as escolhas, as rejeições e as escolhas para chefe. O impacto social, também, correlacionou-se significativamente com as três variáveis das notas $\mathrm{T}(\mathrm{r}=, 744 ; \mathrm{r}=-, 756 ; \mathrm{r}=, 658)$, estas correlações são boas, ou seja, à medida que aumenta o impacto social, aumentam as escolhas, as escolhas para chefe e diminuem as rejeições. Ao correlacionarmos as notas $\mathrm{T}$ das escolhas com o estatuto social (cf. Tabela 7), encontrámos uma relação significativa moderada $(\mathrm{r}=$ ,517). Aqui conseguimos ver que o estatuto social influencia na escolha. No entanto, esta relação não se estende à rejeição e ao chefe.

Tabela 2

Correlação entre a escolha, a rejeição e o chefe e a preferência social, o impacto social e o estatuto social

\begin{tabular}{lllc}
\hline & $\begin{array}{c}\text { Preferência } \\
\text { social }\end{array}$ & $\begin{array}{c}\text { Impacto } \\
\text { social }\end{array}$ & $\begin{array}{c}\text { Estatuto } \\
\text { social }\end{array}$ \\
\hline Escolha &, $602 * *$ &, $744 * *$ &, $517 * *$ \\
Rejeição &, $606 * *$ &,$- 756^{* *}$ & 0,188 \\
Chefe &, $348^{*}$ &, $658^{* *}$ & 0,200 \\
\hline **. A correlação é significativa ao nível 0.01 (2-tailed). \\
*. A correlação é significativa ao nível 0.05 (2-tailed).
\end{tabular}

Após termos feito o teste correlação de Pearson (cf. tabela 3) entre a escolha e os resultados da compreensão emocional (totais das componentes do TEC) não se encontrou nenhuma correlação significativa. Contrariamente à escolha, encontrámos uma correlação significativa entre o total do TEC com a rejeição $(r=$ $-, 315)$, ou seja, à medida que a IE aumenta, diminuem as rejeições; o mesmo não se verificando com o chefe e o total das componentes do TEC. 
Tabela 3

Teste de Correlações entre a escolha, a rejeição e o chefe e o total do TEC

\begin{tabular}{ll}
\hline & TEC \\
\hline Escolha &, 206 \\
Rejeição &,$- 315^{*}$ \\
Chefe &, 136 \\
\hline
\end{tabular}

*A correlação é significativa ao nível 0.05 (2-tailed).

Efetuámos uma correlação de Pearson entre a escolha e todas as componentes do TEC (cf. tabela 4), na qual verificámos, apenas, uma correlação moderada significativa com a regulação emocional (componente 6 do TEC - compreensão da possibilidade de controlar as experiências emocionais) $(\mathrm{r}=, 368)$, isto é, as crianças com maior regulação emocional são as mais escolhidas. Deparamo-nos também com uma correlação significativa moderada entre a nota $\mathrm{t}$ de rejeição e a regulação (componente 6) ( $\mathrm{r}=-, 359)$, quer dizer que as crianças com maior regulação emocional são as menos rejeitadas. De igual modo, verificámos apenas uma correlação significativa moderada entre o chefe e componente 6 do TEC $(r=, 327)$. As crianças que regulam melhor as emoções são as mais escolhidas para líderes.

Tabela 4

Correlações entre: a escolha, a rejeição e o chefe e as nove componentes do TEC

\begin{tabular}{|c|c|c|c|c|c|c|c|c|}
\hline 1 & 2 & 3 & 4 & 5 & 6 & 7 & 8 & 9 \\
\hline $\begin{array}{l}\text { Esco, } 009 \\
\text { lha }\end{array}$ & ,138 & ,102 &,- 067 & ,093 &, $368^{*}$ & 032 & ,035 & ,039 \\
\hline $\begin{array}{l}\text { Rejei -,095 } \\
\text { ção }\end{array}$ &,- 222 &,- 036 &,- 232 &,- 057 &,$- 359^{*}$ & ,043 &,- 100 & ,058 \\
\hline Chefe-,101 &, 164 &,- 018 & 138, &,- 030 &, $327^{*}$ & - 042 & ,036 &,- 040 \\
\hline
\end{tabular}

* A correlação é significativa ao nível 0.05 (2tailed).

Nota: 1- Reconhecimento; 2- Causa Externa; 3- Desejo; 4- Crença;

5- Lembrança; 6- Regulação; 7- Esconder; 8- Misto; 9- Moralidade

Correlacionámos as notas $\mathrm{T}$ de escolha, rejeição e chefe entre si (cf. Tabela 5) e descobrimos que o chefe apresenta correlações significativas com a escolha ( $\mathrm{r}=$ $, 738)$ e a rejeição $(r=-, 315)$, sendo estas boa e moderada, respetivamente. As crianças mais escolhidas ou menos rejeitadas são as escolhidas para chefe.

Tabela 5

Correlação entre as notas $T$ de escolha, rejeição e chefe

\begin{tabular}{|c|c|c|c|}
\hline & Escolha & Rejeição & Chefe \\
\hline Escolha & - &,- 207 &, $738 * *$ \\
\hline Rejeição & - & - &,$- 315^{*}$ \\
\hline Chefe & - & - & - \\
\hline
\end{tabular}

\section{Discussão}

Da influencia das variáveis pessoais, apenas o ter irmãos ou não influencia na escolha do líder, o que vai ao encontro dos resultados de Scharf e Mayseless (2009). Esta variável poderá estar associada a experiências sociais mais comuns e frequentes em casa, o que pode contribuir para uma aprendizagem das crianças de influenciar e gerir outros. A possibilidade de socialização que alguém recebe da sua família poderá estar relacionada com o número de crianças na família e a classificação por idade em comparação com a sua fratria. Irmãos mais novos e crianças em grandes famílias poderão receber maior socialização e maior treino das emoções (Barbera, Christensen, \& Barchard, 2004). Assim, impõe-se falarmos um pouco das competências sociais que aliamos à importância de ter irmãos ou não, pois consideramo-las importantes para a escolha de líderes. Quer parecer-nos que o facto de ter irmãos promove ou poderá promover as competências interpessoais, o que, mais uma vez, nos leva a pensar que esta variável é bastante importante na escolha do líder. Nalguns estudos realizados, encontrámos que as competências interpessoais são de grande valor para a escolha dos líderes infantis. Para Scharf e Mayseless (2009), as crianças que possuem estratégias nas relações interpessoais com os pares, tornam-se competentes nas competências interpessoais pró-sociais que são úteis em grandes grupos. É ainda esperado que os líderes tenham um modelo positivo dos outros e isso inclui preocupar-se com os outros e ter boas aptidões interpessoais.

Verificou-se, igualmente, neste estudo que as crianças em idade pré-escolar que pertenciam ao grupo sociométrico mais alto, eram escolhidos para papéis de liderança com mais regularidade do que os do grupo sociométrico mais baixo. Assim, foi encontrada uma relação positiva entre a escolha para lider e estatuto sociométrico em crianças do pré-escolar, indo ao encontro de vários estudos (Löfdahl, 2006; Fukada et al., 1994, Fukada, Fukada, \& Hicks, 1997). A popularidade, como o focado na literatura (Farmer \& Rodkin, 1996; Hawley, 2002; Pakaslahti \& Keltikangas-Jarvinen, 2001; Rodkin, Farmer, Pearl, \& VanAcker, 2000, Scharf \& Mayseless, 2009), apareceu também como um factor associado à escolha para líder.

Inicialmente, as nossas expectativas eram que a escolha para líder estivesse relacionada com a compreensão emocional e apesar de não termos encontrado correlação significativa com o total das componentes do TEC, encontrámos uma correlação significativa numa das componentes do TEC, a regulação emocional (compreensão das possibilidades de controlar as experiências emocionais). Estes resultados apontam para que a escolha para líder seja feita mais pela capacidade de regular as emoções, do que pela capacidade para expressar ou reconhecer as emoções, ou pelas suas características pessoais. Hensel (1991) reconhece que é importante que as crianças compreendessem os seus próprios sentimentos antes de tentarem entender os dos seus colegas de sala e só depois conseguirem perceber os sentimentos dos outros. $\mathrm{O}$ seu estudo mostra que as crianças que estavam mais despertas para os sentimentos dos colegas, encontravam, com maior frequência, maneiras de ajudar os seus companheiros e, com maior clareza, entender a perspetiva dos outros. Esta visão leva os a crer que as 
crianças terão, também, a sua regulação desenvolvida, o que as fez serem encaradas como líderes pelos seus pares.

As escolas fornecem ambientes naturais para que as crianças aprendam e exercitem as aptidões de liderança e treinem em diferentes estilos de liderança. A experiência que as crianças ganham em posições de liderança nas interações sociais na escola fornece um importante andaime para futuros líderes (Scharf \& Mayseless, 2009).

\section{Referências.}

Barbera, K., Christensen, M., \& Barchard, K. (2004). Relating Family Size and Birth order to emotional intelligence. Paper presented at the Western Psychological Association Annual Convention, Phoenix, Arizona.

Bento, A. (2008). Estilos de liderança dos líderes escolares da Região Autónoma da Madeira. In Costa, J., Neto-Mendes, A. \& Ventura, A. (Org.). Actas do V Simpósio sobre Organização e Gestão Escolar Trabalho Docente e Organizações Educativas, pp.145-157. Aveiro: Universidade de Aveiro. (Publicação em CD-ROM - ISBN 978-972-789-281-5).

Chen, X., Rubin, K. H., Sun, Y. (1992). Social reputation and peer relationships in Chinese and Canadian children: A cross-cultural study.Child Development, 63,1336-1343.

Dodge, K. A. (1983). Behavioral antecedents of peer social status. Child development, 1386-1399.

Farmer, T. W., \& Rodkin, P. C. (1996). Antisocial and prosocial correlates of classroom social positions: The social network centrality perspective. Social Development, 5(2), 174-188.

Fukada, H., Fukada, S., \& Hicks, J. (1997): The Relationship Between Leadership and Sociometric Status Among Preschool Children. The Journal of Genetic Psychology: Research and Theory on Human Development, $158 \quad$ (4), 481-486. doi.org/10.1080/00221329709596684.

Fukada, S., Fukada, H. \& Hicks, J. (1994). Structure of leadership among preschool children. The Journal of Genetic Psychology, 155(4), 389- 395. doi.10.1080/00221325.1994.9914789.

Hawley, P. (2002). Social dominance and prosocial and coercive strategies of resource control in preschoolers. International Journal of Behavioral Development, 26 (2), 167-176.

Hensel, N. H. (1991). Social leadership skills in young children. Roeper Review, 14 (1), 4-6. Retiradode

http://search.ebscohost.com/login.aspx?direct=true \&db $=$ pbh\&AN=9608122561\&1

ang=pt-br\&site $=$ ehost-live.

Kitano, M. K., \& Tafoya, N. (1983). Preschool leadership: A review and critique. Journal for the Education of the Gifted, 5(2), 78-89.

Lee, S. Y., Recchia, S. L., \& Shin, M. S. (2005). Not the Same Kind of Leaders": Four Young Children's Unique Ways of Influencing Others in Journal of
Research in Childhood Education, 20 (2), 132-148. doi.org/10.1080/02568540509594557.

Löfdahl (2006). Grounds for values and attitudes: Children's play and peer-cultures in pre-school. Journal of Early Childhood Research 4(1):77-88.

Mawson, B. (2011). Children's Leadership Strategies in Early Childhood. Journal of Research in Childhood Education, 25 (4), 327-338. doi: 10.1080/02568543.2011.605207.

Morda, R. \& Waniganayake, M. (2010). Emergence of child leadership through play in early childhood. In M. A. Ebbeck \& M. Waniganayake (Eds.), Play in early childhood education: Learning in diverse contexts (pp. 103-118). South Melbourne: Oxford University Press.

Pakaslahti, L., \& Keltikangas-Järvinen, L. (2001). Peer-attributed prosocial behavior among aggressive/preferred, aggressive/non-preferred, non-aggressive/preferred and non-aggressive/non-preferred adolescents. Personality and Individual Differences, 30(6), 903-916.

Popper, M. \& Mayseless, O. (2003). Back to basics: Applying a parenting perspective to transformational leadership. The Leadership Quarterly, 14(1), 41-65.

Popper, M., Amit, K., Gal, R., Mishkal-Sinai, M., \& Lisak, A. (2004). The capacity to lead: Major psychological differences between leaders and non-leaders. Military Psychology, 16 (4), 245-263.

Popper, M., Mayseless, O., \& Castelnovo, O. (2000). Transformational leadership and attachment. Leadership Quarterly, 11, 267-289.

Rechia, S. (2011). Preschool leaders in the early childhood classroom in Murphy, S, \& Reichard, R. (Eds). Early development and leadership. Building the next generation of leaders (pp. 39-58). New York: Taylor \& Francis.

Rodkin, P. C., Farmer, T. W., Pearl, R., \& Van Acker, R. (2000). Heterogeneity of popular boys: antisocial and prosocial configurations. Developmental psychology, 36(1), 14.

Scharf, M. \& Mayseless, O. (2009). Socioemotional Characteristics of Elementary School Children Identified as Exhibiting Social Leadership Qualities. The Journal of Genetic Psychology, 170(1), 73-94. 